\title{
Género, sexualidades y educación. Intersecciones necesarias para una educación emancipadora
}

\author{
Viviana I. Seoane \\ Instituto de Investigaciones en Humanidades y Ciencias Sociales (UNLP-CONICET). Facultad de Humanidadesy \\ Ciencias de la Educación (FaHCE). Universidad Nacional de La Plata (UNLP), Argentina \\ viviseoane@gmail.com \\ Moira Severino \\ Instituto de Investigaciones en Humanidades y Ciencias Sociales (UNLP-CONICET). Facultad de Humanidades y \\ Ciencias de la Educación (FaHCE). Universidad Nacional de La Plata (UNLP), Argentina \\ moiseve988@gmail.com
}

El feminismo, en tanto epistemología crítica, ha hecho una gran contribución al plantear el carácter sexista, androcéntrico y colonial de la ciencia. En su lugar, propone un instrumental teórico capaz de producir otro conocimiento que parta de las historias de mujeres y de aquellos grupos silenciados por la historia y demás disciplinas modernas (Campagnoli, 2018; Curiel, 2009; Lugones, 2010; Quijano, 2000). Las escuelas y el curriculum escolar han cumplido un papel central en la transmisión de un conocimiento que se presenta como universal, ahistórico y neutro, sobre la base de una historia del hombre que no sólo prescindía de las mujeres sino también ignoraba tanto a los varones que no encajaban en el modelo de virilidad hegemónico como a las identidades sexuales que escapaban a la categorización binaria de lo femenino y masculino (Felitti y Queirolo, 2009).

Las escuelas producen un saber sobre los cuerpos de mujeres y varones que se corresponde con lo que Gayle Rubin (1986) denominó sistema sexo/género, y produce subjetivamente a niñxs y jóvenes para asumir cuerpos generizados que respondan a la norma heterocentrada. La escuela reproduce ciertas representaciones de masculinidad y femineidad a través de prácticas discursivas que actúan como tecnologías de género (de Lauretis, 1996). Esta imposición de una norma heterocentrada naturaliza y normaliza la asignación de un género a cada cuerpo sexuado, reivindicando la sola existencia de dos sexos. Como señala de Lauretis, el género no constituye una propiedad de los cuerpos sino la suma de efectos que en ellos se producen, así como en los comportamientos y en las relaciones e interacciones que tiene el sujetx con el mundo exterior. En la escuela tiene lugar una experiencia de género y una serie de "autorepresentaciones producidas en el sujeto por las prácticas socioculturales, los discursos y las instituciones dedicadas a la producción de mujeres y varones" (de Lauretis, 1996, p.26).

La producción en el campo de la educación de investigaciones con perspectiva de género resulta, en los años '80, de la convergencia de dos corrientes de pensamiento: de un lado, el pensamiento feminista y la distinción teórica entre sexo y género muestran el modo en que varones y mujeres viven su escolaridad; del otro, abordajes del campo de la sociología de la educación desde los años sesenta en Francia (Bourdieu y Passeron, 1964; 1970) e Inglaterra (Bernstein, 1989; 1990), rompen con la visión de la escuela como un espacio neutro desprovisto de poder, y lo definen como creador y legitimador de identidades sociales jerarquizadas. 
Ambas corrientes promueven la realización de estudios sobre las instituciones educativas y sobre las formas de transmisión de un pensamiento binario. La intención ya no es constatar las diferencias entre niños y niñas, sino avanzar en propuestas para que la escuela deje de ser sexista. Estos trabajos permitieron construir el mapa de la discriminación sexista ejercida por el sistema educativo y concluir que fue diseñado desde los hombres para la reproducción de la masculinidad.

El análisis microsociológico de las interacciones cotidianas produjo una serie de estudios sobre la posición de las mujeres en el sistema escolar mostrando que existe una desigual distribución de las oportunidades para varones y mujeres. Sin embargo, fue necesario que el feminismo introdujera la categoría género para superar todas aquellas explicaciones de la diferencia sexual basadas en la naturaleza y en una supuesta superioridad masculina. Así, el reconocimiento del carácter social de la diferencia sexual hizo visibles otras huellas de la desigualdad y ofreció nuevas herramientas de análisis para comprender el papel del sistema educativo en la naturalización y reproducción del pensamiento binario en el que se sustenta el patriarcado. La investigación puso el acento en la producción de la femineidad y la masculinidad en y por la escuela, así como la producción de relaciones de subordinación y dominación, basados en los aportes de Michelle Foucault (1992), en particular su caracterización de la microfísica del poder y del modo en que se ejerce en relaciones no igualitarias, así como del carácter positivo-productivo del poder. De este modo, se va consolidando una línea de investigación sobre las relaciones entre género y escuela y sobre el proceso de socialización de las mujeres que, en adelante, permitirá ir develando los modos en que se pone de manifiesto el patriarcado en el campo de la educación (Subirats, 1999).

Las instituciones educativas no sólo reproducen y refuerzan prácticas que afectan las trayectorias escolares y biográficas de aquellxs que no se sienten incluidos en el binarismo y la heterosexualidad. Otras veces, las escuelas producen dispositivos que permiten a lxs niñxs, a lxs jóvenes y a lxs adultxs que habitan las escuelas romper con los estereotipos sociales que fijan las identidades de género y las identidades sexuales. Afirmándonos en una perspectiva constructivista, concebimos la sexualidad como una dimensión de la subjetividad que se construye a lo largo de la vida y no se reduce al conocimiento de los atributos biológicos que diferencian a mujeres de varones. La pregunta acerca de cómo habilitar una enseñanza no sexista recorre las investigaciones que intentan entablar una relación entre los aportes del feminismo y la teoría de género y los que provienen del campo de la educación.

La educación sexual y la pedagogización de la sexualidad infantil y juvenil han sido los términos de mayor disputa acerca de sus sentidos y de quienes se encuentran habilitados a transmitir un saber sobre el cuerpo, la genitalidad, pero también sobre el goce y el deseo. En Argentina, desde la sanción en el año 2006 de la Ley Nacional No26.150 de Educación Sexual Integral (ESI), ha tomado impulso la investigación tendiente a historizar y periodizar los modelos de educación sexual escolar con el propósito de develar las bases teóricas de cada modelo. El modelo dominante ha sido el biologista con la consecuente biologización de prácticas sociales para representar como "anormales" toda combinación de caracteres sexuales que exceda el marco del binarismo sexo/género. Otro modelo dominante es aquél denominado moralizante por priorizar los aspectos vinculares y éticos de la sexualidad sobre la base de un "deber ser" desprovisto de expresiones y sentimientos. Gracias a la perspectiva de género, los estudios sobre masculinidades y la teoría queer, la educación sexual escolar incluye el trabajo sobre "cuestiones de poder que la construcción de la sexualidad implica" y reconoce la existencia de "diversas formas de vivir el propio cuerpo y de construir relaciones afectivas, formas que deben enmarcarse en el respeto por sí mismx y por lxs demás y que merecen -todas- el mismo respeto" (Morgade, 2006, pp.43-44). La ESI se inscribe en un paradigma de derechos humanos y ubica a la sexualidad de niñxs y jóvenes como un derecho tan importante como otros, promueve nuevas visiones y contenidos relativos al derecho a vivir una sexualidad plena y reconoce a niñxs y jóvenes como sujetos sexuados.

En Argentina, como en otros países de América Latina, la actual fase neoliberal produce un constante ataque conservador que desacredita los avances alcanzados en materia de derechos sexuales, así como su expresión curricular en el campo de la educación, y traduce los avances en el campo de una epistemología 
feminista bajo el eufemismo de "ideología de género". Es por ello que se vuelve necesario hacer confluir las luchas de todos aquellos movimientos y organizaciones que defienden los derechos de personas vulneradas por su condición sexual, política y social, junto a la producción que tiene lugar en nuestras universidades públicas y centros de investigación, para que académicxs y militantxs trabajemos en favor de una educación emancipadora.

Los artículos que reúne este dossier nos invitan a reflexionar sobre los desafíos que atraviesan, en el presente, el abordaje de los géneros y las sexualidades en las escuelas. Aunque los textos pueden leerse de manera aleatoria, aquí proponemos un recorrido que parta del trabajo de Guacira Lopes Louro, originalmente publicado por Vozes en el año 2003, en el que aborda los géneros y las sexualidades a partir del reconocimiento de diversas identidades sexo/genéricas en la escuela, y analiza sus efectos en instituciones en las que tradicionalmente ha imperado una lógica normalizadora. Las "identidades excéntricas" -que podríamos describirlas también como identidades no binarias- poseen el potencial de cuestionar el centro y su carácter de construido, de ficcional. Analiza las formas en que la constitución de las diferencias y las identidades de género y sexuales se han expresado en el campo del curriculum, y destaca que los cuestionamientos de diferentes feminismos, del movimiento negro y de la disidencia sexual a la ausencia de sus historias en el curriculum oficial no lograron interpelar el canon escolar. Frente a esto, Lopes Louro elabora una estrategia desestabilizadora que analice y problematice las formas a través de las cuales se construyen las diferencias, las clasificaciones y jerarquías, e interpela a lxs educadorxs a asumir los riesgos de la precariedad, las dudas y la provisoriedad de las respuestas a sabiendas de que no existen soluciones definitivas. El artículo de Lopes Louro recobra relevancia en el actual contexto político de Brasil porque son las "identidades excéntricas" sobre las que se cierne un ataque conservador machista, sexista, homofóbico, para volver "normal" lo que hace poco fuera reconocido por la sociedad y por el sistema educativo como posible y equivalente.

Proponemos, a continuación, un salto a la sección "Intervención polémica" de este número para leer a Graciela Morgade, quien nos invita a pensar la ESI como un proyecto de justicia social en tanto aporta a la emancipación de las mujeres y otrxs grupos oprimidxs. Para ello, es imprescindible que académicxs, investigadorxs y docentes realicemos una crítica epistemológica a los saberes de referencia que componen el curriculum que nos permita recuperar los sentimientos y las experiencias como fuente del conocimiento, y visibilizar aquello que estuvo históricamente silenciado. El reconocimiento del carácter sexuado de la educación constituye un acto de justicia política y social. En su texto, Morgade reflexiona sobre la noción de la "construcción social del cuerpo sexuado", la cual enmarca en tres grandes organizaciones estructurales de poder: el patriarcado heteronormativo homo/lesbo/bitransfóbico, el capitalismo y la colonialidad. Cada uno de estos vectores no puede ser analizado por separado. Reconoce que la ESI, en tanto política pública, traza un camino de interpelación de estas estructuras aun cuando presenta vacíos epistemológicos sobre los que nos invita a pensar. Si bien reconoce la potencia que posee la ESI para despatriarcalizar la educación, Morgade propone seguir trabajando en la incorporación de otras y todas las dimensiones de la sexualidad, entre ellas el placer. Sostenida en una perspectiva de género y una ética de los derechos humanos, la ESI aporta al reconocimiento de las personas como sujetos de derechos y sujetos de deseo. Una tarea central y un desafío para lxs docentxs es reconceptualizar la categoría cuerpo para romper con una mirada binaria y esencialista de los sexos/géneros, transversalizar la ESI en todas las dimensiones de la práctica educativa, recuperar los aportes de la pedagogía feminista y reconocer que la ESI es más que una política pública, es, en definitiva, un proyecto político de justicia social.

Seguidamente, la lectura del artículo de Iván Molina Jiménez nos acerca un fragmento de la historia de la educación sexual en Costa Rica a través de un abordaje por los diferentes actores e intereses en pugna y una mirada puesta en el papel de las educadoras en dicho proceso. En su artículo, Molina Jiménez analiza la educación sexual en Costa Rica entre las décadas de 1920 y 1960 utilizando como fuentes principales periódicos y memorias de los Ministerios de Educación y de Salubridad. Allí describe que en su etapa inicial la educación sexual en el Costa Rica de la década de 1920 estaba dominada por varones - esto es médicos y 
jerarcas educativos- y sus contenidos orientados a la higiene sexual cuyo propósito predominante era advertir a los varones sobre los peligros de las enfermedades venéreas. Sin embargo, el autor explica cómo algunas educadoras comenzaron a construir sus propias posiciones de autoridad en dicha materia, dentro y fuera de los espacios oficiales, y la enseñanza de la educación sexual alcanzó también a las mujeres, comenzando a incorporarse un discurso científico sobre la maternidad. Otra etapa inicia en 1930 cuando la educación sexual desborda los límites del aula y alcanza diversos sectores sociales, incluidas las clases trabajadores. Junto a ello, surge una demanda específica de parte del movimiento estudiantil para que la educación sexual se incorpore a los planes de estudio. Molina Jiménez indaga cómo la influencia creciente de la Iglesia católica en el sistema educativo, luego de 1940, llevó a que la educación sexual, tras perder espacio en el Ministerio de Educación, fuera liderada por la cartera de Salubridad, a cuyas diversas actividades se sumaron maestras y profesoras para promover la enseñanza de la sexualidad. Dicha cartera, a la vez que dejaba atrás los enfoques eugenésicos, empezó a desarrollar un amplio programa de enseñanza sobre la sexualidad basado en folletos, publicaciones en los periódicos, charlas y exhibiciones cinematográficas. Si bien Molina Jiménez expresa que falta investigar más a fondo el papel jugado por las educadoras en la enseñanza sexual durante las décadas de 1940 y 1950 , plantea que la información por ahora disponible sugiere que se mantuvieron activas en ese campo y que fueron aliadas de las autoridades de salud constituyendo una resistencia secular frente a la influencia creciente de la iglesia católica en el sistema educativo.

El trabajo de Pablo Scharagrodsky, Carolina Ojeda y Santiago Zemaitis propone una reflexión sobre los fundamentos modernos de la educación escolar de la sexualidad desde la lente de la pedagogía queer, que dialoga con los estudios de género y la pedagogía feminista, para poner en cuestión las prácticas educativas que, en relación con los géneros y las sexualidades, son producidas y naturalizadas por el discurso escolar. En una clara apuesta de lxs autorxs, el trabajo denota un esfuerzo por decontruirlas visiones sobre las identidades, los cuerpos y los placeres que han primado en un orden pedagógico tradicional, heteronormativo y patriarcal. En este ensayo, Scharagrodsky, Ojeda y Zemaitis, a partir de intercambios y discusiones con estudiantes suscitadas en un proyecto de extensión universitaria, sistematizaron las diferentes ideas, representaciones y opiniones de lxs mismxs sobre el lugar de los cuerpos, los géneros y las sexualidades, e identificaron seis problemáticas o tensiones naturalizadas en torno a la educación de la sexualidad. Dichas problemáticas, en torno a los cuales se/nos interrogan, son la heteronorma y la definición de una economía del deseo normal, el cuerpo como dato biológico y universal que produjo la pedagogía moderna, el silencio en torno de la homosexualidad, la reproducción como único fin de la sexualidad y, por último, la pedagogización de la sexualidad y la mirada adultocéntrica de la sexualidad. En este ejercicio de deconstrucción de las visiones sobre las identidades, los cuerpos y los placeres que han primado en un orden pedagógico tradicional, heteronormativo y patriarcal, se reconocen otras voces, que no forman parte del canon académico ni educativo, voces de las calles, de la disidencia, consignas políticas, performances y otras expresiones culturales y políticas, en un intento por operar pedagógicamente sobre los fundamentos modernos de la educación escolar de la sexualidad. Frente a la pregunta ¿qué es lo educable en la educación sexual? Scharagrodsky, Ojeda y Zemaitis reconocen que la sexualidad no puede pensarse como un campo de experiencias fijo y estable, de allí la imposibilidad de que la sexualidad sea una dimensión a ser enseñada. Frente a esto lxs autorxs apuestan por una educación sexual en revisión permanente, inestable, contingente, precaria, atenta a las demandas de su tiempo y a lxs sujetos de la educación, en tanto sujetos históricos y políticos. En este camino recuperan el carácter cuestionador e interrogativo de la pedagogía queer, como así también la potencia trasformadora y liberadora de la pedagogía feminista y una pedagogía del cuidado que enseñe sobre la autonomía corporal y la ciudadanía sexual más plena, para que los encuentros sexuales, eróticos y/o afectivos con otrxs sean más igualitarios, libres y placenteros.

El recorrido propuesto por este dossier nos acerca al trabajo de val flores y su pregunta ¿es la práctica pedagógica una práctica sexual? A partir de tres experiencias de taller de formación, la autora busca problematizar la fuerza heterosexualizante de la cultura escolar y considerar la potencia erótica de los procesos 
de producción del conocimiento. Los talleres disparan un pensamiento que la autora caracteriza como dis/torsionado por preguntas intolerables para el régimen de inteligibilidad de la normalidad educativa y que provocan un radical extrañamiento en los constructos y los órdenes conceptuales de las sexualidades y en nuestros imaginarios pedagógicos y eróticos. Desde una posición feminista prosexo y al ritmo de una pedagogía antinormativa/cuir, flores nos invita a pensar en las terminologías que se nos imponen para pensar educativamente la ESI, los límites de lo pensable, las ignorancias, los residuos o silencios que producen ciertos discursos sobre ella. Ignorancia aquí no implica falta de conocimiento sino el efecto de un conocimiento hegemónico, esto es, una forma heterosexualizante del conocer. Reflexiona sobre cómo la escuela y sus especialistas construyen en las docentes un des-apasionamiento por los modos de pensar interrogativos, por las prácticas de escritura y lectura sin fines instrumentales, además de los efectos políticos y afectivos para nuestra autonomía intelectual. En diálogo con otrxs autorxs, val flores introduce conceptos que atravesaron los talleres como: erotismo colonial, justicia erótica, eroticidad y propone "lo apropiado" como el modo de pensar las sexualidades y cuerpos en la escuela. La autora propone construir un pensamiento pedagógico antinormativo para deshigienizar la educación sexual, desmedicalizarla, despedagogizarla, desescolarizarla, es decir, descolonizarla de sus sentidos más normativos. La pregunta ¿es la práctica pedagógica una práctica sexual? en una invitación a pensar, entre otras cosas, acerca del propio trabajo como docentes, el lugar del cuerpo en la enseñanza, las prácticas pedagógicas normalizadoras, los posicionamientos políticos ante las sexualidades, los géneros y deseos, y en la construcción artesanal de herramientas de intervención e implicación corporal y el diseño de cartografías de los condicionantes y posibilidades institucionales para hacer posible otra pedagogía.

Por último, el recorrido concluye en la lectura de dos investigaciones que operacionalizan las categorías género y sexualidades en ámbitos educativos. Por un parte, Verónica Millenaar aborda las expectativas de formación y sentidos asignados al trabajo por parte de varones egresados de Centros de Formación Profesional (FP), con el objetivo de complejizar el rol de dichas instituciones como dispositivos de socialización ocupacional y de género. El artículo presenta resultados de investigación y se basa en 30 entrevistas realizadas a jóvenes de entre 18 y 32 años durante los años 2018 y 2013. A partir de la sistematización del material recogido y del análisis de los discursos recogidos en las entrevistas, la autora presenta tres grupos de varones en los que confluyen las expectativas de formación, los sentidos asignados al trabajo y las imágenes de sí mismos como varones y trabajadores para producir diferentes modelos de masculinidad. En el primer grupo, el trabajo es la esfera que adquiere centralidad en sus trayectorias, en el segundo grupo las redes de sociabilidad barrial y en el tercer grupo el estudio. Este análisis microsociológico permite a la autora mostrar una diversidad de experiencias de masculinidad que participan de la FP, y los sentidos heterogéneos que se construyen en relación con la formación. Como comenta Millenaar conocer dicha diversidad es un modo de complejizar el rol de estos dispositivos en relación a la socialización ocupacional y de género, y contribuir a desnaturalizar las desigualdades de género que aún persisten en el sistema educativo.

Por otra parte, Talita Gonçalves Medeiros analiza las representaciones sobre la/s lesbianidad/es producidas por estudiantes mujeres de una escuela de la región sur del estado de Rio Grande do Sul. La investigación que sirve de base al artículo tuvo como objetivo interrogar y comprender las representaciones sobre la o las lesbianas producidas por cinco estudiantes mujeres de entre 18 y 20 años de una escuela agrícola de la región sur del estado de Rio Grande do Sul. Orientada por los Estudios Culturales Lesbofeministas, la autora obtuvo sus datos a partir de "ruedas de conversaciones" con las estudiantes mujeres. En el ámbito escolar, la "lesbianidad/es" se encuentra atravesada por la invisibilidad histórico-escolar y cuando es visible, se ancla en representaciones mediadas solamente por la violencia. Los espacios de reflexión sobre la situación femenina y más específicamente sobre la lesbianidad no son frecuentes en la escuela, y cuando se abordan son marcados por un currículo prescrito, cerrado y duro basado en una lógica heterosexual. De esta manera, la lesbianidad se configura como una categoría visible sólo en los espacios de interacción extra-clase de la escuela. Al no 
promover la equidad entre los géneros e ignorar las multiplicidades performáticas de las sexualidades, la escuela cristaliza la heteronormatividad y no contribuye a la extinción de las situaciones de violencia contra las lesbianas manteniéndolas bajo el velo de la invisibilidad. De la conversación con las jóvenes, la autora concluye que la institución educativa todavía se muestra conservadora en lo que se refiere a los patrones sociales a ser seguidos por hombres y mujeres. Este proceso está interpelado por el silenciamiento que la escuela produce y por las normas y reglas que priman en las familias y que, en muchos casos, refuerzan la agresión. Estas situaciones acaban por reverberar en los prejuicios y límites impuestos a las representaciones sobre las lesbianidades.

\section{REFERENCIAS}

Bernstein, B. (1989). Clases, códigos y control I. Estudios teóricos para una sociología del lenguaje. Madrid: Akal.

Bernstein, B. (1990). La construcción social del discurso pedagógico. Bogotá: El Griot.

Bourdieu, P. et Passeron , J. C. (1964). Les étudiants et la culture. París: Les Héritiers.

Bourdieu, P. et Passeron, J. C. (1970). La reproduction. Eléments pour une théorie du système d'enseignement. París: Les Editions Minuit.

Campagnoli, M. (2018). Epistemologías críticas feministas. Aproximaciones actuales. Descentrada: revista interdisciplinaria de feminismos y género, 2 (2), e047. Recuperado de http://www.descentrada.fahce.unlp.edu.a r/article/view/DESe047

Curiel, O. (2009). Descolonizando el feminismo: una perspectiva desde América Latina y el Caribe. En Primer Coloquio Latinoamericano sobre Praxis y Pensamiento Feminista, Buenos Aires, Argentina. Recuperado de ht tp://www.bdigital.unal.edu.co/39749/

de Lauretis, T. (1996). La tecnología del género. Revista Mora, 2, 6-34.

Felitti, K. y Queirolo, G. (2009). Historia. Cuerpos, género y sexualidades a través del tiempo(pp. 27-58). En S. Elizalde, K. Felitti y G. Queirolo (coordinadoras), Género y sexualidades en las tramas del saber. Revisiones y propuestas. Buenos Aires: Libros del Zorzal.

Foucault, M. (1992). Microfisica del poder. Madrid: La Piqueta.

Lugones, M. (2016). Hacia un feminismo descolonial. La manzana de la discordia, 6(2), 105-117. Recuperado de htt p://revistas.univalle.edu.co/index.php/la_manzana_de_la_discordia/article/view/1504

Morgade, G. (2006). Educación en la sexualidad desde el enfoque de género. Una antigua deuda de la escuela. Novedades educativas, 184, 40-44.

Quijano, A. (2000). Colonialidad del poder, eurocentrismo y América Latina (pp. 122- 151). En E. Lander (Ed.) (2000), La colonialidad del saber: eurocentrismo y ciencias sociales. Perspectivas latinoamericanas. Buenos Aires: CLACSO.

Rubin, G. (1986). El tráfico de mujeres: notas sobre la “economía política del sexo”. Nueva antropología, 8(30), 95-145.

Subirats, M. (1999). Género y escuela (pp. 19-31). En C. Lomas (ed.), ¿Iguales o diferentes? Género, diferencia sexual, lenguaje y educación. Madrid: Paidós. 